\title{
Dire le vrai dans la première modernité. Langue, esthétique, doctrine, dir. Dominique de Courcelles
}

Concetta Cavallini

\section{OpenEdition}

\section{Journals}

Electronic version

URL: http://journals.openedition.org/crm/13964

DOI: $10.4000 / \mathrm{crm} .13964$

ISSN: 2273-0893

\section{Publisher}

Classiques Garnier

\section{Electronic reference}

Concetta Cavallini, «Dire le vrai dans la première modernité. Langue, esthétique, doctrine, dir. Dominique de Courcelles », Cahiers de recherches médiévales et humanistes [Online], Comptes-rendus, Online since 28 September 2016, connection on 15 October 2020. URL : http://journals.openedition.org/crm/13964 ; DOI : https://doi.org/10.4000/crm.13964

This text was automatically generated on 15 October 2020 .

(c) Cahiers de recherches médiévales et humanistes 


\title{
Dire le vrai dans la première modernité. Langue, esthétique, doctrine, dir. Dominique de Courcelles
}

\author{
Concetta Cavallini
}

\section{REFERENCES}

Dire le vrai dans la première modernité. Langue, esthétique, doctrine, dir. Dominique de Courcelles, Paris, Classiques Garnier, 2014, 266 p.

ISBN 978-2-8124-1234-9

1 La question de l'énonciation de la vérité à l'époque moderne est une question qui fonde le partage du sens et de la parole sociale entre les $\mathrm{XV}^{\mathrm{e}}$ et XVII ${ }^{\mathrm{e}}$ siècles. C'est l'époque que veut analyser l'ensemble des études qui constitue ce volume : il ne s'agit pas de définir la vérité, «mais plutôt de s'interroger sur les différentes modalités d'énonciation du vrai à la naissance de l'époque moderne»(p. 7). Le volume se compose de quatre parties, chacune essayant de focaliser l'attention sur un des aspects les plus saillants de la problématique de « dire le vrai ». La première partie (« Langue et vérité », p. 13 et suiv.) pose les bases de la réflexion abordant le rôle de la langue dans la constitution d'une sorte de « canon » de la vérité. Dans le multilinguisme propre à la période allant $\mathrm{du} \mathrm{XV}^{\mathrm{e}}$ au XVII ${ }^{\mathrm{e}}$ siècle, y a-t-il une langue qui, plus qu'une autre, est censée véhiculer la vérité ? Il suffit de rappeler qu'aux langues qui, comme le latin et le grec, sont en honneur en raison du prestige de la culture classique, il faut aussi ajouter l'hébreu dont l'importance procède des Écritures ; il convient finalement de prendre en compte aussi les différentes langues vulgaires qui, pour des raisons politiques, s'affirment de plus en plus en Europe. Les trois premières études analysent des cas emblématiques : Érasme (A. Vanautgaerden) chez qui l'amour pour la vérité et l'opposition à la calomnie, « véritable danger pour l'État » (p. 80), portaient souvent à 
un langage impulsif et apparemment violent ; La Ramée (M.-D. Couzinet), foncièrement pédagogue, chez qui la vérité de langage coïncidait souvent avec l'explication philologique des textes classiques, ou avec leur traduction du latin et du grec. Une troisième étude concerne la problématique de l'exégèse biblique fondée sur la connaissance de l'hébreu (hebraica veritas) dans la Salamanque du XVI siècle ( $\mathrm{F}$. Dominguez Reboiras).

2 La deuxième partie ("Esthétique, la belle énonciation du vrai», p. 87 et suiv.) fait porter l'accent sur la coïncidence entre beauté et vérité à travers deux études qui analysent la beauté des gravures allégoriques qui illustrent la Civitas Veri sive Morum de Bartolomeo Del Bene (R. Gorris Camos) et la place accordée aux poètes dans le Commentaire au livre de la Sagesse de R. Holkot, dominicain anglais ayant vécu au milieu du XIV ${ }^{e}$ siècle (E. Babey). L'analyse est d'autant plus remarquable que, si l'on est attentif aux mentalités, jamais un homme du Moyen Âge s'interrogeant sur le vrai n'aurait pris en considération des poètes, comme Holkot l'a fait.

3 La troisième partie ("L'écriture de l'histoire : entre esthétique et doctrine », p. 155) concerne l'historiographie et son influence dans le domaine du politique à travers l'importance que les règles d'énonciation revêtent, dans la première modernité, pour les princes et leurs États. Les deux études, très rigoureuses, se concentrent sur la réalité espagnole, analysant la Cronica abreviada de España de Diego de Valera (1482) (S. LòpezRìos et C. Moya Garcia) et le Tratado des descubrimiento de las Indias y de la conquista de Juan Suarez de Peralta (E. Gonzàles Gonzàlez). Dans ces derniers cas, la vérité historique ne peut qu'être subjective, les auteurs adoptant souvent un point de vue personnel. Parfois ce point de vue rapporte la vision de leur maître, d'autres fois c'est bien un point de vue personnel mais muable; il ne faut pas oublier que la participation aux événements d'une époque dense de conflits et de contrastes pouvait déterminer un changement de faction et donc de point de vue. Il faut s'interroger alors sur ce qui est "crédible» et ce qui est "véritable» (p. 204) en faisant ressortir une ambiguïté possible dans l'interprétation du texte de ces œuvres (p. 181).

4 La dernière partie s'interroge sur l'élément peut-être le plus complexe de tout le volume : le rapport entre vérité et doctrine, ce dernier mot ayant besoin d'être redéfini à l'intérieur du volume. En effet, deux axes de l'analyse ont besoin d'être combinés : « le souci de faire doctrine dans les textes normatifs de l'âge classique et le devenir de la doctrine dans les textes et les comportements non doctrinaux» (p.11). Les histoires narrées par les mystiques espagnols se présentent comme une preuve de contre-vérité à l'égard de la doctrine officielle (D. de Courcelles) : dans ces histoires la doctrine est "balbutiée ", non dite (p. 227) comme preuve de manque de savoir et d'humilité. Le vrai se superpose à la culture et aux traditions dans l'analyse du rapport entre vérité de la foi et place des images dans les manifestations de cultures religieuses (cultes, processions, etc.) (E. Bermès). Les histoires de la philosophie aux XVII ${ }^{e}$ et XVIII siècles se posent aussi le problème du vrai (U. J. Schneider), conscientes du fait que le terme "doctrine " a chez elles un statut ambigu, puisqu'il désigne presque un élément extérieur à la discipline elle-même.

5 Chaque étude est complétée par une bibliographie attentive et précise, qui ajoute à l'utilité et à la précision de l'ensemble. Le sujet est trop vaste pour être analysé de manière complète dans un seul volume; les articles, sans rechercher l'exhaustivité, présentent avec rigueur et clarté des pistes intéressantes et des exemples qu'on ne peut pas ignorer lorsqu'on se pose la question du statut de l'énonciation de la vérité dans la 
première modernité. La perspective transdisciplinaire des études, ainsi que la rigueur des développements font de cet ouvrage un précieux instrument de référence pour les chercheurs. 